\title{
Calculating the Detector Solid Angle in X-ray Energy Dispersive Spectroscopy
}

Nestor J. Zaluzec

Electron Microscopy Center, Materials Science Div, Argonne National Laboratory, Argonne IL

The detector solid angle $(\Omega)$ in X-ray Energy Dispersive Spectroscopy (XEDS) is one of the important parameters controlling our ability to measure signal generated by the electron solid interaction, however, it is often overlooked or incorrectly calculated. In order to optimize detection of elemental species it is incumbent upon the analyst to maximize its value. In addition, with the new generation of silicon drift detectors a range of alternate geometries is possible in electron columns and due to their compact size these detectors can potentially achieve high geometrical collection efficiencies. Traditional solid angle approximations break down in some of these geometries, and we present formulae herein which can be used to more precisely determine its value.

The conventional method for calculating the nominal solid angle of a detector is to compare its active detection area to the equivalent surface area of a sphere nominally located at the same radial distance from the specimen. To calculate the solid angle $(\boldsymbol{\Omega})$ one simply takes the surface area $(\boldsymbol{S})$ of the equivalent sphere divided by the square of its radius $(\boldsymbol{R}): \Omega=\mathrm{S} / \mathrm{R}^{2}$. While this is an exact equation, the typical approximation made in the microanalysis community is to replace $\boldsymbol{S}$ by $\boldsymbol{A}$ the active area of the detector which is collecting the x-ray signal and $\boldsymbol{R}$ by $\boldsymbol{d}$, the radial specimen-todetector distance (figure 1). This approximation rapidly leads to non-physical values ( $>2 \pi)$ as $\boldsymbol{A}$ increases and $\boldsymbol{d}$ decreases. For a detector whose active area is circular in cross-section, or whose active area is defined by a circular collimator it can be shown that appropriate solid angle formulae can be written as [1]:

$$
\Omega=\frac{S}{R^{2}}=\frac{2 \pi\left(a^{2}+d^{2}-d \sqrt{a^{2}+d^{2}}\right)}{a^{2}+d^{2}}
$$

This relationship is plotted in figure 2 for detectors of active area from $10-100 \mathrm{~mm}^{2}$, the asymptote is for which is $2 \pi$ sterradians. Here $\boldsymbol{a}$ is the radius of the equivalent detector active area and $\boldsymbol{d}$ the radial detector to specimen distance. In order to use this relationship, it is essential to know the actual value of $\boldsymbol{d}$, which in many cases is also ill-defined or not accurately determined. Fortunately the value of $\boldsymbol{d}$ can be experimentally determined by a simple procedure outlined herein.

Under constant electron optical operating conditions it can be shown that the Intensity $(I)$ of a given X-ray line will vary simply as $I=\kappa * \boldsymbol{\Omega}=\boldsymbol{\kappa} * \boldsymbol{S} / \boldsymbol{R}^{2}$ here, $\kappa$ is a term which incorporates all the physics associated with $\mathrm{x}$-ray generation, absorption and fluorescence processes in the specimen. Taking the logarithm of both sides of this equation results in the relationship:

$$
\ln (I)=\ln (\kappa * S)-2 \ln (R)
$$

This is equation is in the form of a simple linear relation $y=b+m x$, where we can associate the slope $m=-2$ and the intercept $b=\ln (\kappa * S)$. Many $\mathrm{x}$-ray detectors are mounted on instruments with linear translation mechanisms allowing an analyst to insert/remove the detector along a radial line of sight path to the specimen. While these mechanisms allow precise linear motion, their disadvantage is that the exact end-point value is not accurately known. To determine the value of $\boldsymbol{R}_{\boldsymbol{o}}$, the fully 
inserted minimum distance of a detector in this geometry, one simply measures $\boldsymbol{I}$ for various values of $\boldsymbol{R}(\boldsymbol{\delta} \boldsymbol{R})$ and plots the result on a $\log -\log$ plot as shown in figure 3. A plot of this form will be linear, however only a single solution will exist whose slope $=-2$. Thus the determination of $\boldsymbol{R}_{\boldsymbol{o}}$ is a simple iterative process. One begins by assuming an initial value of $\boldsymbol{R}_{\boldsymbol{o}}$ and uses the incremental displacement of the detector $\boldsymbol{\delta} \boldsymbol{R}$ to determine the range of values of $\boldsymbol{R}(\boldsymbol{\delta} \boldsymbol{R})$. The data is plotted and the best linear fit is found. In general, the slope of this curve will differ from the value of -2 . The same data set is then recalculated by changing the initial value of $\boldsymbol{R}_{\boldsymbol{o}}$ and replotted and a new best fit is iteratively obtained. Each incremental change of the starting point $\boldsymbol{R}_{\boldsymbol{o}}$ will gradually change the calculated intercept and slope. This process is continued until one sweeps through the value where the slope is -2 . The value of $\boldsymbol{R}_{\boldsymbol{o}}$ at that point represents the best estimate of the distance $\boldsymbol{d}$ $=\sqrt{R_{o}^{2}-a^{2}}$, which should be used in all subsequent solid angle calculations.

\section{References}

[1] Zaluzec, N.J. "Detector Solid Angle Calculations for X-ray Energy Dispersive Spectrometry" Microsc. Microanal. 15, 93-98, 2009 doi:10.1017/S1431927609090217

[2] This work was supported by the US DOE Office of Science, Contract DE-AC02-06CH11357 at ANL
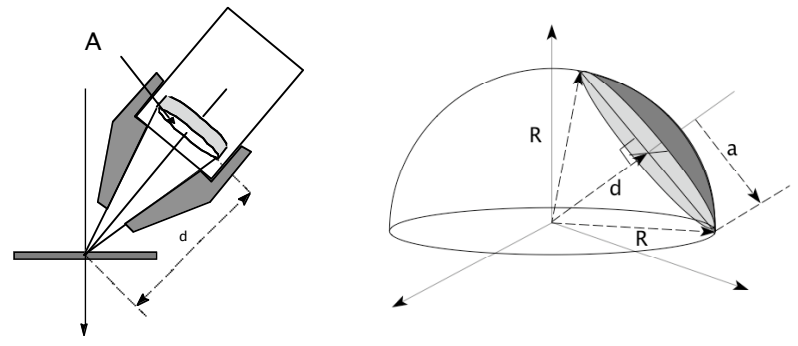

Fig 1.)

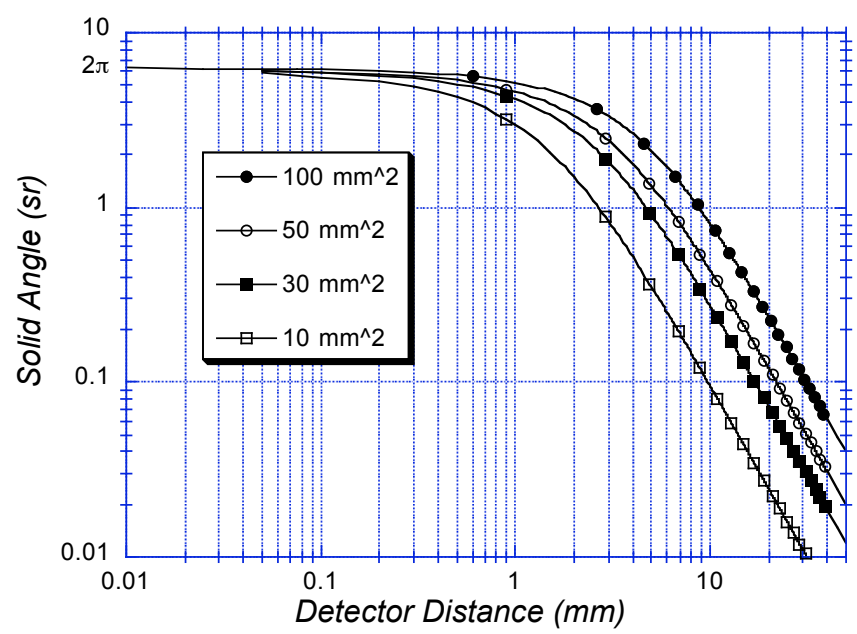

Fig 2.)
Figure 1. The geometry of a solid angle calculations detector active area (A) is represented by a plane section of circular cross-section defined by its radius $\boldsymbol{a}$ which is positioned at a distance $\boldsymbol{d}$ from the specimen. $\boldsymbol{R}$ is the effective radius of the subtending sphere.

Figure 2. Calculated solid angle for various active area detectors at a range of distances from the specimen.

Figure 3. Log-Log plot of $\ln (\boldsymbol{I})$ vs $\ln (\boldsymbol{R})$ to determine the best experimental value of $\boldsymbol{R}_{\boldsymbol{o}}=4.12 \mathrm{~mm} @ \mathrm{~m}=-2$

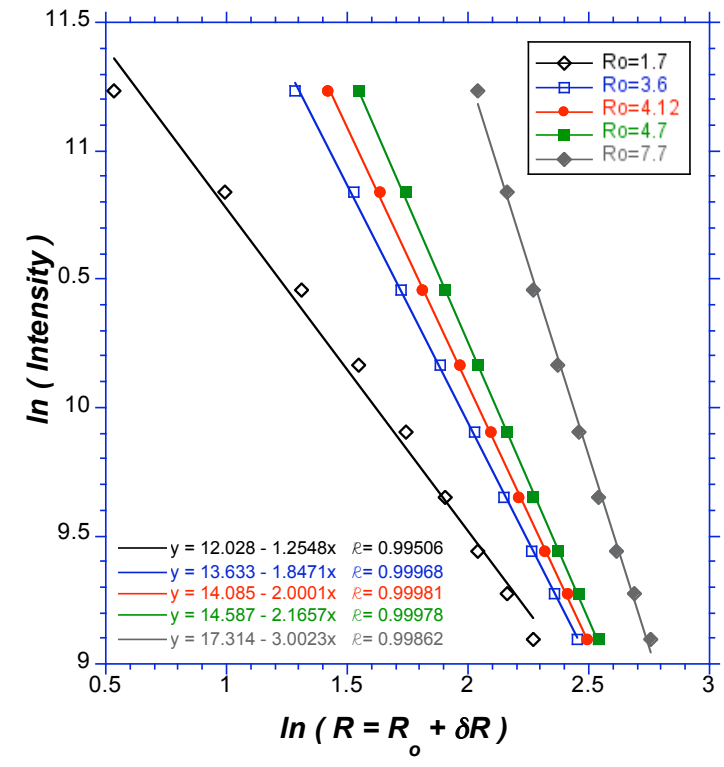

Fig 3.) 